\title{
Agenesia del Tercer Molar en una Etnia Originaria del Norte de Chile: Atacameños o Lican Antai
}

\author{
Third Molar Agenesis in Native Ethnia from North of Chile: Atacameños or Lican Antai \\ *Fernando García-Hernández \& **Víctor Javier Beltrán Varas
}

GARCÍA-HERNÁNDEZ, F. \& BELTRÁN, V. V. J. Agenesia del tercer molar en una etnia originaria del Norte de Chile: Atacameños o Lican Antai. Int. J. Morphol., 26(3):583-590, 2008.

RESUMEN: Agenesia es la ausencia de dientes por alteraciones genéticas aisladas o sindrómicas. La agenesia del tercer molar está asociada a malformaciones y considerada por diversos autores consecuencia de la evolución humana (Larmour et al., 2005). Son los dientes con mayor prevalencia de agenesia junto a los segundos premolares e incisivos laterales (Fuller \& Denehy, 1984). La prevalencia varía entre 9 y 37\% (McNamara \& Foley, 2006), en tanto, Arboleda et al. (2006) señalan una prevalencia del 20\%. La literatura señala variables estadísticas porcentuales, por género, por arcada dentaria, por lado y por diente, con escasos artículos sobre grupos originarios de Chile. La población en estudio consistió en 33 hombres y 57 mujeres de 16 a 55 años, de la etnia atacameña, sin exodoncias del tercer molar ni tratamientos ortodónticos y sin malformaciones congénitas. Se determinó el grado de mestizaje mediante técnica serológica de hemo-aglutinación y por aplicación de la fórmula de Bernstein, que demostró 56\% de mezcla indígena. A cada individuo se le tomó radiografía panorámica para observar presencia o ausencia de terceros molares. Se determina un 26,7\% de individuos con agenesia de uno o más terceros molares, con mayor porcentaje en hombres. En la muestra y en hombres hay mayor agenesia de terceros molares mandibulares; en cambio, en mujeres existe mayor agenesia de terceros molares maxilares. Predominan agenesias izquierdas, lo mismo se comprueba en mujeres, mientras en hombres se comprueba igual porcentaje bilateral. Predomina la agenesia de dos molares en ambos sexos. No existen diferencias estadísticas significativas al $95 \%$ y los resultados coinciden con la literatura. La investigación representa un aporte a la antropología del Norte de Chile, pero considerando lo reducido de la muestra, no fue posible determinar variables étnicas.

PALABRAS CLAVE: Antropología; Odontología; Agenesia; Tercer molar.

\section{INTRODUCCIÓN}

La agenesia o anodoncia parcial es la ausencia congénita de dientes asociada a síndromes, desórdenes genéticos únicos o aislada sin componente genético, donde falta uno o más dientes de la dentición. El concepto de hipodoncia corresponde a la ausencia congénita de menos de 6 dientes, más compleja y que incluye alteraciones en forma, número, posición y período eruptivo, mientras que oligodoncia establece la ausencia de 6 o más dientes aparte de los terceros molares (Vastardis, 2000; Klein et al., 2005). Algunos autores consideran que la ausencia de terceros molares no debe considerarse como agenesia sino como parte del proceso evolutivo (Larmour et al., 2005).

Jorgenson (1980) lo señala como un carácter poligénico hereditario, que es observable en miembros de una misma familia (Woelfel \& Scheid, 1998). Vastardis in- forma sobre la identificación de genes alterados responsables de la no formación de terceros molares. Kolenc (2004), Klein et al.; Briceño et al. (2006) y Tallón-Walton et al. (2007) consideran que, como probable causa genética de agenesia familiar en molares, sería la mutación de los genes MSX1 y PAX9.

Es conocida la teoría de la reducción terminal dentaria de Adloff (citado por Figún \& Garino, 1992) que señala la futura desaparición del tercer molar en la especie humana, aspecto evolutivo consecuencia de la disminución de la actividad masticatoria. Es una línea evolutiva hacia un número menor de dientes (Shafer et al., 1977). En 2001, Proffit citado por Arboleda et al. (2006), indica que las tendencias evolutivas han influido en la dentición actual y en la dimensión de los maxilares, en tanto que Bailit (1975) sugiere que

\footnotetext{
* Unidad de Anatomía, Departamento Biomédico, Facultad de Ciencias de la Salud, Universidad de Antofagasta, Antofagasta, Chile.

*** Cirujano Dentista.
} 
la fórmula dentaria futura podría excluir a incisivos laterales, segundos premolares y terceros molares.

Según señalaron Figún \& Garino, la evolución de los hábitos alimenticios ha contribuido a modificar el tamaño de los maxilares, siendo marcado este cambio a nivel mandibular, aspecto evolutivo del homo sapiens sapiens que se confirma por la disminución progresiva del espacio retromolar entre borde anterior de la rama y cara distal del tercer molar, lo que actualmente se determina mediante la clasificación de Pell y Gregory (que mide el espacio que tiene el tercer molar para erupcionar entre borde anterior de rama y cara distal del segundo molar (Sandhu \& Kaur, 2005).

Sin embargo, autores como Rozhkova et al. (1999) sugieren que "la agenesia no debe considerarse como una manifestación de la reducción filogenética del número de dientes sino como una anomalía del desarrollo producto de un proceso de mutación y selección basado en la herencia".

La causa de esta patología se debe a una alteración durante el proceso de formación de la lámina dentaria, que comienza entre el quinto mes de vida intrauterina y el nacimiento.

La agenesia compromete a varios dientes de la dentición humana, pero la literatura señala que son los terceros molares los que demuestran una mayor prevalencia (Fuller \& Denehy, 1984; Woelfel \& Scheid; Lozada, 2003; Bartolomé, 1993; Sarmiento \& Herrera, 2004), siendo el orden habitual de los dientes ausentes el siguiente: terceros molares, premolares maxilares y mandibulares e incisivos laterales (Fuller \& Denehy).

Al respecto, la revisión de la literatura muestra una multiplicidad de trabajos referidos a la agenesia dentaria en general, muchos de los cuales excluyen a los terceros molares por su gran variabilidad.
Nanda (1954) señalaba que en la literatura no existe uniformidad en la recolección de datos y en la interpretación de los resultados respecto de agenesias en terceros molares, situación que actualmente se mantiene y que podemos confirmar luego de revisar más de un centenar de publicaciones, lo que dificulta la comparación objetiva de los resultados, aunque es el diente con mayor prevalencia de agenesias.

Existe una gran variabilidad en los datos respecto de las diferencias de género, en el número de agenesias, en la arcada y lado predominante, en los porcentajes de agenesia, etc. McNamara \& Foley (2006) señalan que la hipodoncia del tercer molar tiene una prevalencia entre $9 \%$ y $37 \%$, mientras Arboleda et al. señalan una prevalencia de agenesia poblacional del $20 \%$, coincidiendo en que existe una mayor frecuencia en mujeres respecto de hombres, con una relación de 3:2.

Siendo el tercer molar la pieza dentaria que provoca mayores patologías asociadas con su ausencia, retención, erupción y variabilidad de posición una vez erupcionada y cuyo síntoma principal es el dolor que produce, así como las dificultades quirúrgicas durante su exodoncia y las complicaciones postquirúrgicas (Cauvi \& Feldman, 1989; Ahlqwist \& Gröndahl, 1991; Feldman et al., 1995; Martínez et al., 1995; Punwutikorn et al., 1999), efectuamos un estudio anatomo radiológico para determinar el grado de agenesia de terceros molares, según casos detectados y número total de terceros molares, su variabilidad según género, arcada y lado predominante y su relación etnoantropológica, en una población originaria del Norte de Chile, que corresponde al pueblo atacameño o lican antai, grupo étnico constituido según el censo 2002 (INE Instituto Nacional de Estadísticas de Chile, 2002) por 21.015 individuos, que habla un idioma propio llamado kunza y que ocupa una extensa área andina y altiplánica de la Segunda Región, entre los 2.400 y 3.600 metros s.n.m. (Bustos, 1999; Bustos \& Lehnert, 2000).

Los resultados de nuestra investigación se comparan con los obtenidos por diversos autores y en especial de América Latina y de Chile.

Tabla I. Tipificación sistema ABO en individuos atacameños según factor Rh (n=90).

\begin{tabular}{lcclcc}
\hline Factor Rh+ & $\mathbf{N}^{\mathbf{0}}$ casos & Porcentaje & Factor Rh- & $\mathbf{N}^{\mathbf{0}}$ casos & Porcentaje \\
\hline Grupo AB & 0 & 0 & Grupo AB & 0 & 0 \\
Grupo A & 21 & 23,33 & Grupo A & 0 & 0 \\
Grupo B & 3 & 3,33 & Grupo B & 0 & 0 \\
Grupo 0 & 63 & 70,00 & Grupo 0 & 3 & 3,33 \\
\hline Total & 87 & 96,66 & Total & 3 & 3,33 \\
\hline
\end{tabular}




\section{MATERIAL Y MÉTODO}

Para cumplir con los aspectos bioéticos en la realización de la investigación, se solicitó a cada individuo participante su consentimiento informado, autorizando realizar examenes clínico y radiográfico de cavidad oral completa mediante radiografía panorámica y examen de sangre para determinar grupo sanguíneo y factor Rh para comprobar el grado de mestizaje.

Se analizó una muestra dirigida de 90 individuos de la etnia atacameña, de ambos sexos, sin malformaciones hereditarias y, a lo cuales, no se les hubiese efectuado ningún tratamiento de ortodoncia ni exodoncias de los terceros molares, determinando su origen en base a los apellidos paterno y materno según documentación señalada por la Corporación Nacional de Desarrollo Indígena CONADI y en bibliografía antropológica de la zona, obtenida en la base de datos del Instituto de Investigaciones Antropológicas de la Universidad de Antofagasta o por aporte de investigadores, y determinando su grado mestizaje mediante técnica serológica de hemoaglutinación con placas utilizando sueros testigos (Tabla I).

El grado de mestizaje indígena que presenta la población atacameña fue establecida en base a la frecuencia génica de los alelos $\mathrm{O}$ y d(Rh-) del grupo sanguíneo y del factor $\mathrm{Rh}$, aplicando la fórmula de Berstein: $\mathrm{M}=(\mathrm{Pm}-\mathrm{Pe}) /$ (Pi- Pe), donde:

M: grado de mestizaje.

Pe: frecuencia génica en la población española.

Pi: frecuencia génica en la población indígena.

Pm: frecuencia génica en la población mixta.

La frecuencia génica de la población española fue determinada por Campillo, mientras que la frecuencia génica indoamericana fue comprobada por Matson et al., ambos autores citados por Reyes (2005).

La comprobación de la agenesia se efectuó mediante examen clínico intraoral y radiografías panorámicas completas. Las películas usadas fueron de marca Kodak, se empleó equipo radiográfico modelo ARCOGRAPH ZEUS RF producido por IMAGO Radiology S.R.L. de Italia y se usó reveladora automática alemana marca DÜRR modelo XR25 Typ 1702-04.
Las características del grupo de individuos en estudio se detallan en la Tabla II.

\section{RESULTADOS}

El grado de mestizaje indígena que presenta la muestra de población atacameña se estableció en base a frecuencia génica de los alelos $\mathrm{O}$ y d(Rh-) del grupo sanguíneo y del factor Rh, aplicando la fórmula de Berstein se aprecia en la Tabla III y que presenta un alto grado de mestizaje producto de la ausencia de un 70\% de los alelos A y B (Grupo O) y de la escasa presencia del alelo d (Rh-) con 3,33\%.

Tabla II. Características de la muestra.

\begin{tabular}{lllll}
\hline Género & n & Edad & Rango edad & \% \\
\hline Masculino & 33 & 26,6 & $18-55$ años & 36,7 \\
Femenino & 57 & 25,6 & $16-39$ años & 63,3 \\
Total & 90 & 25,9 & $16-55$ años & 100,0 \\
\hline
\end{tabular}

La agenesia determinada para la muestra atacameña se detalla en las Tablas IV a IX.

\section{DISCUSIÓN}

El diagnóstico de agenesia del tercer molar es recomendable realizarlo después de los 14 años de edad, a causa de posible calcificación tardía, coincidiendo con autores como Llarena del Rosario \& Nuño González (1990), Bartolomé et al. (1994), Uzamis et al. (2000) y Bolaños et al. (2003), ya que existen múltiples trabajos en que se inicia el análisis radiográfico en edades muy tempranas y, como expresan claramente Tavajoni-Kermani et al. (2002), el tercer molar debe considerarse agenésico cuando está ausente después de 14 o más años de edad que, de acuerdo a lo que señalan Peck et al. (1996), correspondería a la "edad crítica" para observar la formación del tercer molar.

Del análisis de nuestros resultados es posible deducir lo siguiente respecto a: porcentaje de individuos que pre-

Tabla III. Frecuencia génica de los alelos $\mathrm{O}$ y d ( $\mathrm{Rh}-)$ en individuos atacameños.

\begin{tabular}{cccc}
\hline & Alelo $\mathbf{O}$ & Alelo d $($ Rh-) & Mezcla Indígena \% \\
\cline { 2 - 4 } Frecuencia génica $(\mathrm{n}=90)$ & 0,856 & 0,163 & $56 \%$ \\
\hline
\end{tabular}


Tabla IV. Distribución porcentual de agenesia de terceros molares en atacameños $(n=90)$. *No hay diferencias estadísticamente significativas según género al $95 \%$.

\begin{tabular}{lcclcc}
\hline \multicolumn{2}{l}{ Agenesia según casos. $\mathbf{n = 9 0}$} & \multicolumn{4}{l}{ Agenesia terceros molares $\mathbf{n = 3 6 0}$} \\
\hline Género & $\mathrm{n}$ & $\%$ & Género y n de agenesias & $\mathrm{n}$ & $\%$ \\
\hline Masculino & $9 / 33$ & 27,3 & Masculino & $12 / 132$ & $9,09 *$ \\
Femenino & $15 / 57$ & 26,3 & Femenino & $33 / 228$ & 14,47 \\
Total & $24 / 90$ & 26,7 & Total & $45 / 360$ & 12,50 \\
\hline
\end{tabular}

Tabla V. Agenesia de terceros molares en atacameños según arcada y número total de terceros molares. *No hay diferencias estadísticamente significativas al 95\% según género y arcada.

\begin{tabular}{lcccc}
\hline \multirow{2}{*}{ Género } & \multicolumn{2}{c}{ Maxilar } & \multicolumn{2}{c}{ Mandíbula } \\
\cline { 2 - 5 } & $\mathrm{n}$ & $\%$ & $\mathrm{n}$ & $\%$ \\
\hline Masculino & $3 / 12$ & $25,0^{*}$ & $9 / 12$ & $75,0^{*}$ \\
Femenino & $18 / 33$ & 54,5 & $15 / 33$ & 45,5 \\
Total & $21 / 45$ & 46,7 & $24 / 45$ & 53,3 \\
\hline
\end{tabular}

Tabla VI. Distribución porcentual de atacameños con agenesia según tercer molar ausente de la arcada.

\begin{tabular}{|c|c|c|c|c|c|c|c|}
\hline \multicolumn{4}{|c|}{ Maxilar } & \multicolumn{4}{|c|}{ Mandíbula } \\
\hline \multicolumn{2}{|c|}{ Diente 1.8} & \multicolumn{2}{|c|}{ Diente 2.8} & \multicolumn{2}{|c|}{ Diente 4.8} & \multicolumn{2}{|c|}{ Diente 3.8} \\
\hline $\mathrm{n}$ & $\%$ & $\mathrm{n}$ & $\%$ & $\mathrm{n}$ & $\%$ & $\mathrm{n}$ & $\%$ \\
\hline $6 / 45$ & 13,3 & $15 / 45$ & 33,3 & $12 / 45$ & 26,7 & $12 / 5$ & 26,7 \\
\hline
\end{tabular}

Tabla VII. Distribución porcentual de agenesia terceros molares en atacameños, según lado y género. *No hay diferencias estadísticamente significativas al 95\% según lado predominante.

\begin{tabular}{|c|c|c|c|c|c|c|c|}
\hline \multicolumn{4}{|c|}{ Lado derecho } & \multicolumn{4}{|c|}{ Lado izquierdo } \\
\hline \multicolumn{2}{|c|}{ Masculino } & \multicolumn{2}{|c|}{ Femenino } & \multicolumn{2}{|c|}{ Masculino } & \multicolumn{2}{|c|}{ Femenino } \\
\hline $\mathrm{n}$ & $\%$ & $\mathrm{n}$ & $\%$ & $\mathrm{n}$ & $\%$ & $\mathrm{n}$ & $\%$ \\
\hline $6 / 12$ & 50 & $12 / 33$ & 36,4 & $6 / 12$ & 50 & $21 / 33$ & 63,6 \\
\hline \multicolumn{4}{|c|}{ Total } & \multicolumn{4}{|c|}{ Total } \\
\hline \multicolumn{2}{|c|}{$\mathrm{n}$ agenesias } & \multicolumn{2}{|c|}{$\%$} & \multicolumn{2}{|c|}{$\mathrm{n}$ agenesias } & \multicolumn{2}{|c|}{$\%$} \\
\hline \multicolumn{2}{|c|}{$18 / 45$} & \multicolumn{2}{|c|}{40} & \multicolumn{2}{|c|}{$27 / 45$} & \multicolumn{2}{|c|}{60} \\
\hline
\end{tabular}

Tabla VIII. Distribución porcentual de agenesia de uno o más terceros molares en atacameños, según número de dientes ausentes y género.

\begin{tabular}{lcccccc}
\hline Agenesia/Género & \multicolumn{2}{c}{ Masculino } & \multicolumn{2}{c}{ Femenino } & \multicolumn{2}{c}{ Total } \\
\hline \multirow{2}{*}{ Molar } & $\mathrm{n}$ & $\%$ & $\mathrm{n}$ & $\%$ & $\mathrm{n}$ & $\%$ \\
2 Molares & 3 & 20 & 6 & 66,7 & 9 & 37,5 \\
3 Molares & 9 & 60 & 3 & 33,3 & 12 & 50,0 \\
4 Molares & 0 & 0 & 0 & 0 & 0 & 0 \\
Total de casos agenesia & 3 & 20 & 0 & 0 & 3 & 12,5 \\
\hline
\end{tabular}

Tabla IX. Distribución de agenesia por género según, número de terceros molares ausentes en atacameños.

\begin{tabular}{lll}
\hline Masculino & Femenino & Total \\
\hline $1-2-4$ & $2-4-1$ & $2-1-4$ \\
\hline
\end{tabular}


sentan uno o más terceros molares con agenesia: El porcentaje de $26,7 \%$ de individuos con agenesia de terceros molares obtenido en nuestro trabajo se encuentra dentro del rango de $20 \%-30 \%$ determinado por autores como Keene $(1965)=27 \%$; Ortega Piga $(1987)=29 \%$; Shad \& Boyd (1979)=23,3\%; Thorson \& Häag (1991)=23\% y Bredy et al. $(1991)=20,7$, todos citados por Bartolomé; Thompson et al. (1974)=22,3\%; Hattab (1995)=27,0\%; Lynham $(1990)=22,7 \%$; Mok \& Ho $(1996)=28,5 \%$; Rozhkova et al. $=22,5 \%$; Kruger et al. $(2001)=27,9 \%$, Sandhu \& Kaur $=24,0 \%$, a nivel latinoamericano con los obtenidos en trabajos realizados por Cuairán et al. $(1996)=26,75 \%$ y Silva Meza $(2003)=24,3 \%$ en mexicanos; Sarmiento \& Herrera $=21,3 \%$ en colombianos; Castilho et al. $(1990)=20,39 \%$; Paula \& Ferrer $(2007)=20,18$ en brasileños.

Porcentajes menores a $20 \%$ fueron obtenidos por Garn et al. $(1963)=16,4 \%$, citado por Bartolomé; y a nivel de Latinoamérica, Crispin et al. (1972)=16,0\%; Nicodemo $(1973)=18,5 \%$ y Oliveira \& Serra Negra $(1976)=14,42 \%$ todos citados por Bartolomé, además de Oliveira \& Serra Negra $(1984)=10,83 \%$ en brasileños, Henríquez (1972) $=15,0 \%$ citado por Bartolomé y Méndez \& Contreras $(2006)=15,36 \%$ en venezolanos; Altunaga et al. $(2008)=12,45 \%$ en cubanos; Bastidas \& Rodríguez $(2004)=10,6 \%$ en colombianos.

Mientras que porcentajes por sobre el 30\% fueron determinados por Goren et al. (2005)=38,5\%; Bartolomé $=30,8$; Lima de Castro et al . (2006) $=40,13 \%$ en brasileños y Feldman et al. $=44,75 \%$ en Chile.

En la Tabla X se muestran los autores que determinaron porcentajes en que existe diferencia estadísticamente significativa al $\mathrm{p}<0,05$, respecto del porcentaje obtenido en nuestra investigación.

La literatura muestra rangos entre 3,75\% y 29,89\% de agenesia respecto del total de terceros molares, obteniendo en nuestro trabajo un valor intermedio de $12,5 \%$ de agenesia.

En relación al porcentaje de agenesia de terceros molares respecto del número total de dientes que debieran estar presentes en la dentición humana adulta, la literatura señala rangos entre $1,70 \%$ y $19,4 \%$, estando nuestros resultados muy cercanos al rango menor al determinarse un 3,33\% de agenesia.

Predomina la agenesia en el género masculino $(27,3 \%-26,3 \%)$, sin diferencias estadísticas significativa al $95 \%$, coincidiendo con lo citado en la literatura ya que aunque en diversas investigaciones existe predominio de uno $\mathrm{u}$ otro género, se confirma que en la mayoría de los casos no existe diferencia estadística significativa al $95 \%$ de confiabilidad.

La agenesia mandibular predomina en todo el grupo en estudio $(53,4 \%)$ y en hombres $(75,0 \%)$; en cambio en mujeres existe mayor agenesia maxilar $(46,7 \%)$, sin diferencias estadísticas significativa al $95 \%$ de confiabilidad según género y arcada. Los valores son coincidentes con lo citados en la literatura.

Predominan los casos de agenesia izquierda (60\%), con marcada diferencia a lo citado en la literatura que señala un mayor número de casos de agenesia derecha en relación a los terceros molares. Lo mismo se comprueba en mujeres $(63,6 \%)$, mientras que los hombres presentan iguales porcentajes en ambos lados.

Tabla X. Comparación de porcentajes de agenesia del tercer molar determinados por diversos investigadores que muestran diferencias estadísticamente significativas al 95\% (p<0,05) respecto de los valores obtenidos en nuestra investigación $(26,7 \%$-n=90).

\begin{tabular}{llcccl}
\hline Autor(es) & \multicolumn{1}{c}{ País } & Año & n & \% & Diferencia estadística p<0,05 \\
\hline Crispim, J. et al. & Brasil & 1972 & 490 & 16,0 & $\mathrm{p}<0,0144161$ \\
Henríquez, P.E. & Venezuela & 1972 & 200 & 15,0 & $\mathrm{p}<0,0179125$ \\
Oliveira, O. L. \& Serra Negra, E. & Brasil & 1976 & 416 & 14,42 & $\mathrm{p}<0,00453076$ \\
Oliveira, O. L. \& Serra Negra, E. & Brasil & 1984 & 120 & 10,83 & $\mathrm{p}<0,0028645$ \\
Altunaga, A. et al. & Cuba & 2003 & 265 & 12,45 & $\mathrm{p}<0,001468$ \\
Bastidas, M.A. \& Rodríguez, A.M. & Colombia & 2004 & 141 & 10,6 & $\mathrm{p}<0,00144119$ \\
Méndez, D.C. \& Contreras, Ch.C. & Venezuela & 2006 & 397 & 15,36 & $\mathrm{p}<0,0105005$ \\
Feldman, I. et al. & Chile & 1995 & 143 & 44,75 & $\mathrm{p}<0,00565889$ \\
Goren, S. et al. & Israel & 2005 & 226 & 38,5 & $\mathrm{p}<0,0473651$ \\
Lima de Castro, E.V.F. et al. & Brasil & 2006 & 224 & 40,13 & $\mathrm{p}<0,0252131$ \\
\hline
\end{tabular}


En la muestra en estudio el tercer molar superior izquierdo (2.8.) es la pieza dentaria que muestra el mayor porcentaje de agenesia, con un $33,3 \%$.

En nuesto estudio se detectó un mayor número de casos con doble agenesia, con un 50\%; mientras que el porcentaje de agenesias simples fue de $37,5 \%$, coincidiendo ambos valores con lo citado en la literatura, que indica el predominio de las agenesias simples o dobles. No se detectaron casos de triple agenesia.

\section{CONCLUSIONES}

Para el diagnóstico y estudios evolutivos sobre la ausencia o presencia del tercer molar, los exámenes radiográficos deben realizarse a individuos mayores de 14 años de edad.
Se comprueban variables porcentuales notables en diferentes trabajos referidos a la agenesia del tercer molar, algunos muy cercanos a nuestro estudio y otros con variaciones porcentuales menores o mayores, siendo algunas de estas diferencias porcentuales estadísticamente significativas (Tabla $\mathrm{X}$ ).

La muestra atacameña presenta agenesia de los terceros molares en un 26,7\% de los casos, predominando la agenesia en el género masculino, a nivel mandibular, en el lado izquierdo, de tipo doble y siendo la pieza dentaria prevalente el tercer molar superior izquierdo, sin presentarse diferencias estadísticas significativas al 95\% de confianza.

La presente investigación es un aporte al estudio antropológico de las culturas del Norte de Chile, pero debido a lo reducido de la muestra no permite determinar variables étnicas específicas.

GARCÍA-HERNÁNDEZ, F. \& BELTRÁN, V. V. J. Third molar agenesis in native ethnia from North of Chile: Atacameños o Lican Antai. Int. J. Morphol., 26(3):583-590, 2008.

SUMMARY: Agenesis is the absence of teeth by genetic alterations, single or as syndrome. Agenesis of third molar is associated to malformations and is considered by diverse authors a consequence of the human evolution (Larmour et al., 2005). The third molars together with second premolars and lateral incisors are the teeth with greater prevalence of agenesis (Fuller \& Denehy, 1984). The prevalence varies between 9\% and 37\% (McNamara \& Foley, 2006); Arboleda et al. (2006) indicated a prevalence of 20\%. Literature indicate variable percentage, by gender, dental arches, side and tooth, with few articles on original groups of Chile. The population in study consisted of 33 men and 57 women between 16 and 55 years of the ethnic group of atacameños, without extractions of third molar nor orthodontic treatments and without congenital malformations. Hybridism was determined by means of serum technique by blood agglutination and by application of the formula of Bernstein, demonstrated a $56 \%$ of indigenous mixture. To each individual a panoramic x-ray was taken to observe presence or absence of third molars. A $26.7 \%$ of individuals with agenesis of one or more third molars was determined, with greater percentage among males. Agenesis lower third molar predominates in the sample and in men; however in women are greater agenesis upper third molar. In addition, agenesis predominates of the left side in both sexes, while in men equal bilateral percentage is verified. Agenesis of two molars predominates in both sexes. Statistical analyses did not show significant differences at the $95 \%$ level, and the results, in general, agree with those in the literature. This research represents a contribution to the anthropology of the north of Chile, but it is not possible to determine ethnic variables considering the small sample in study.

KEY WORDS: Anthropology; Dentistry; Agenesis; Third molar

\section{REFERENCIAS BIBLIOGRÁFICAS}

Ahlqwist, M. \& Gröndahl, H. G. Prevalence of impacted teeth and associated pathology in middle-age and older Swedish women. Community Dent. Oral Epidemiol. 19:116-9, 1991.

Altunaga, A.; Hidalgo, A.; Burgos, G. \& Puig, L. La oligodoncia un problema a tener en cuenta. http:// www.monografías.com/trabajos 28/oligodoncia/ oligodoncia.shtml, 2008.

Arboleda, L. A.; Echeverri, J; Restrepo, L. A ; Marín, M. L.; Vásquez, G.; Gómez, J. C.; Manco, H. A.; Pérez, C. M \&
Taborda, E. Agenesia dental. Revisión bibliográfica y reporte de dos casos clínicos. Rev. Fac. Odontol. Univ. Antioq., 18(1):47-54, 2006.

Bailit, H. L. Dental variation among population. An anthropologic view. Dent. Clin. North Am., 19(1):12539, 1975.

Bartolomé, B. Desarrollo y agenesia del tercer molar en una población de niños y adolescentes españoles. Tesis Doctoral. Universidad Complutense de Madrid, 1993. http:// www.cibernetia.com/tesis_es/CIENCIAS_MEDICAS/ 
CIRUGÍA/ESTOMATOLOGIA_Y_ORTODONCIA/22 (fecha de acceso 10 de Noviembre de 2007).

Bartolomé, B.; Barbería, E.; De Nova, J. \& Mourelle, M. R. Deasarrollo del tercer molar en una muestra de niños y adolescentes españoles I. Odontol. Pediatr., 3:62-9, 1994.

Bastidas, M. A. \& Rodríguez, A. M. Agenesia dental en pacientes jóvenes. Rev. Estomatología, 12(2):34-43, 2004.

Briceño, I.; Berrocal, M. C.; González, O. A.; Gutiérrez, S. J.; Ordóñez, A. \& Torres, D. Mecanismo de herencia de la agenesia dental. http://www.encolombia.com/odontología/investigaciones/memorias-Vencuentroanalisis.htm., 2006.

Bolaños, M. V; Moussa, H.; Manrique, M. C. \& Bolaños, M. J. Radiographic evaluation of third molar development in Spanish children and young people. Forensic Sci. Int., 133(3):212-9, 2003.

Bustos, A. Etnografía Atacameña. $1^{\text {a }}$ ed. Antofagasta, Editorial Universidad de Antofagasta, 1999. pp.57-92.

Bustos, A. \& Lehnert, R. Historia del pueblo atacameño. $1^{\text {a }}$ ed. Antofagasta, Fundación Minera Escondida, 2000.

Cauvi, D. \& Feldman, I. Presencia clínica de los terceros molares y su relación con la pérdida de los primeros y/o segundos molares permanentes. Rev. Iberoamericana de Ort., 9(1):9-25, 1989.

Castilho, J. C. M.; Nicodemo, R. A.; Bazzarella, C. B \& Moraes, L. C. Prevâlencia de anodontia entre estudiantes do $2^{\circ}$ Grau da cidade de Säo José dos Campos: correlacäo dessa anomalia entre terceiros molares e outros órgäo. Rev. Odontol. UNESP, 19(1):269-76, 1990.

Corporación Nacional de Desarrollo Indígena CONADI. http:/ /www.conadi.cl/index.html

Crispim, J.; Trigueiro, K. G.; De Sá e Benavides Filho, F. R. \& Salzano, F. M. Third molar agenesis in a trihybrid Brazilian population. Am. J. Phys. Anthrop., 37(2):289-92, 1972.

Cuairán, V.; Gaitán, L. A. \& Hernández, A. J. Agenesia dental en una muestra de pacientes ortodónticos del Hospital Infantil de México. Rev. ADM, 53(4):211-5, 1996.

Feldman, I.; Estupiñán, C.; Cauvi, D.; Espinoza, A. \& Rajecic, Z. Estudio comparado de agenesia de terceros molares en pacientes figurados y no figurados. Rev. Fac. Odont. Univ. de Chile, 13(1):26-35, 1995.
Figún, M. E. \& Garino, R. R. Anatomía odontológica funcional y aplicada. $2^{\mathrm{a}}$ ed. $4^{\mathrm{a}}$ Reimp. Buenos Aires, El Ateneo, 1992. pp.341-2.

Fuller, J. L. \& Denehy, G. E. Concise dental anatomy and morphology. Chicago, Year Book Publishers, 1984. pp.264-5.

Goren, S.; Tzoisner, R.; Dinbar, A.; Levin, L. \& Brezniak, N. Prevalence of congenitally missing teeth in Israeli recruits. Refuat Hapeh Vehashinaim 22(2):49-53, 2005.

Hattab, F. N. Impaction status of third molars in Jordanian students. Oral Surg. Oral Med. Oral Pathol. Oral Radiol. Endond., 79(1):24-9, 1995.

Instituto Nacional de Estadísticas INE. Estadísticas Sociales de los Pueblos Indígenas de Chile Censo 2002. $1^{\mathrm{a}} \mathrm{ed}$. Santiago, INE, 2002.

Jorgenson, R. J. Clinician's view of hypodoncia. J. Am. Dent. Assoc., 101:283-6, 1980.

Klein, M. L.; Nieminen, P.; Lammi, L.; Niebuhr, E. \& Kreiborg, S. Novel mutation of the initiation codon of PAX9 causes oligodontia. J. Dent. Res., 84(1):43-7, 2005.

Kolenc, F. J. Agenesias dentarias: en busca de alteraciones genéticas responsables de la falta de desarrollo. Med. Oral Patol. Oral Cir. Bucal, 9(5):385-95, 2004.

Kruger, E.; Thompson, W. M. \& Konthasinghe, P. Third molar outcomes from age 18 to 26: Findings from populationbased New Zealand longitudinal study. Oral Surg. Oral Med. Oral Pathol. Oral Radiol. Endod., 92(2):150-5, 2001.

Larmour, C. J.; Mossey, P. A; Thind, B. S.; Forgie, A. H.; Stirrups, D. R. \& Colin, J. Hypodontia- a retrospective review of prevalence and etiology. Part I. Quintessence Int., 36:263-70, 2005.

Lima de Castro, E. V. F.; Lima de Castro, A.; Pescinini, L. M.; Coelho, P. T: \& Biazon, A. T. Agenesia e inclusao dental patológica. Estudo clínico e radiográfico em pacientes. Rev. Fac. Odontol. Lins, Piracicaba, 18(1):41-6, 2006.

Llarena del Rosario, M. E \& Nuño González, M. M. Etapas de formación y calcificación del tercer molar. Rev. ADM, 47:112-7, 1990.

Lozada, O. Alteraciones en el número de dientes asociados a maloclusiones. Tesis de Postgrado Ortodoncia, Facultad de Odontología, Universidad Central de Venezuela, 2003. 
Lynham, A. Ranoramic radiographic survey of hipodoncia in Australian Defence Force recruits. Aust. Dent. J., 35(1):19-22, 1990.

Martínez, S.; Concha, G. \& San Pedro, J. Estudio radiográfico de terceros molares inferiores en una muestra de individuos de 18 a 20 años. Rev. Fac. Odont. Univ. de Chile, 13(1):43-9, 1995.

McNamara, C. \& Foley T. Multidisciplinary management of hypodontia in adolescents: case report. J. Can. Dent. Assoc., 72(8):740-6, 2006.

Méndez, D. C. \& Contreras, Ch. C. Anomalías de múmero en pacientes con dentición mixta. http://www.ortodoncia.ws/ publicaciones/2006/anomalias_numero _pacientes_denticion_mixta.asp\#.

Mok, Y. Y. \& Ho, K. K. Congenitally absent third molars in 12 to 16 year old Singaporean Chinese patients: a retrospective radiographic study. Ann. Acad. Med. Singapore, 25(6):828-30, 1996.

Nanda, R. S. Agenesis of the third molar in man. Am. J. Orthod., 40:698-706, 1954.

Oliveira, O. L. \& Serra Negra, E. Agenesia de terceiros molares em negros brasileiros. Arch. Cent. Est. Fac. Odont. Univ. Fed. M. Gerais, 21/22(2/1):103-11, 1984.

Paula, A. F. B. \& Ferrer, K. J. N. Prevalência de agenesia em uma clínica ortodôntica de Goiânia. $R G O, 55(2): 149-53$, 2007.

Peck, S.; Peck, L. \& Kataja, M. Site-specificity of tooth agenesis in subjects with maxillary canine malpositions. Angle Orthod., 66(6):473-6.

Punwutikorn, J.; Waikakul, A. \& Ochareon, P. Synptoms of unerupted mandibular third molar. Oral Surg. Oral Med. Oral Pathol. Oral Radiol. Endod., 87(3):305-10, 1999.

Reyes, C. Parámetros de crecimiento facial en niños mapuche. Santiago de Chile, Facultad de Odontología, Universidad de Chile, 2005.

Rozhkova, E.; Markova, M. \& Dolefsi, J. Studies on agenesis of third molar among populations of different origins. $S b$. Lek., 100(2):71-84, 1999.

Sandhu S. \& Kaur, T. Radiographic evaluation of the status of the third molar in the Asian-Indian students. J. Oral Maxilofacial Surg., 63(5):640-45, 2005.
Sarmiento, P. \& Herrera, A. Agenesia de terceros molares en estudiantes de Odontología de la Universidad del Valle entre 16 y 25 años. Colomb. Med., 35:(3):5-9, 2004.

Shafer, W.; Hine, M. \& Levy, B. A textbook of Oral Pathology. $4^{\mathrm{a} e d .}$ Philadelphia, W.B. Saunders Company, 1977.

Silva Meza, R. Radiographic assessment of congenitally missing teeth in orthodontic patients. Int. J. Paediatr. Dent. 13(2):112-6, 2003.

Tallón-Walton, V.; Manzanares-Céspedes, M. C.; Sirpa, A.; Carvalho-Lobato, P; Valdivia-Gandur, I.; GarcíaSusperregui, A.; Ventura, F. \& Nieminen, P. Identification of a novel mutation in the PAX9 gene in a family affected by oligodontia and other dental anomalies. Eur. J. Oral Sci., 115:427-32, 2007.

Tavajoni-Kermani, H.; Kapur, R. \& Sciote, J. J. Tooth agenesis and craniofacial morphology in an orthodontic population. Am. J. Orthod Dentofacial Orthop., 122(1):39-47, 2002.

Thompson, G. W.; Popovich, F. \& Anderson, D. L. Third molar agenesis in the Burlington Growth Centre in Toronto. Community Dent. Oral Epidemiol., 2(4):187-92, 1974.

Uzamis, M.; Kansu, Ö.; Taner, T. U. \& Alpar, R. Radiographic evaluation of the third-molar development in a group of Turkish childrens. J. Dent. Child., 67:136-41, 2000.

Vastardis, H. The genetics of human tooth agenesis: new discoveries for understanding dental anomalies. Am. J. Orthod. Dentofacial Orthop., 117:650-6, 2000.

Woelfel, J. B. \& Scheid, R. C. Anatomía Dental. Aplicaciones Clínicas. Barcelona, Masson-Williams \& Wilkins España, S.A. 1998.

Dirección para correspondencia:

Prof. Dr. Fernando García-Hernández

Unidad de Anatomía, Departamento Biomédico

Facultad de Ciencias de la Salud

Universidad de Antofagasta

Coquimbo 945, Depto 64

Antofagasta

CHILE

Recibido : 10-05-2008

Aceptado: 25-07-2008 